\title{
КЛИНИЧЕСКАЯ ХАРАКТЕРИСТИКА И ИСХОДЫ ЛЕЧЕНИЯ ПАЦИЕНТОВ С АКТГ- ЭКТОПИРОВАННЫМ СИНДРОМОМ
}

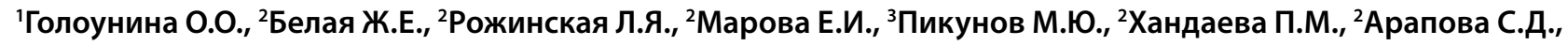
${ }^{2}$ Дзеранова Л.К., ${ }^{2}$ Кузнецов Н.С., ${ }^{1}$ Фадеев В.В., ${ }^{2}$ Мельниченко Г.А., ${ }^{2}$ Дедов И.И.

1 ФГАОУ ВО «Первый Московский государственный медицинский университет им. И.М. Сеченова» Минздрава России (Сеченовский Университет), Москва

2 ФГБУ «Национальный медицинский исследовательский центр эндокринологии» Минздрава России, Москва ${ }^{3}$ ФГБУ «Национальный медицинский исследовательский центр хирургии им. А.В. Вишневского» Минздрава России, Москва

ЦЕЛЬ: изучить клинико-лабораторные характеристики, провести анализ результатов хирургического лечения и длительного динамического наблюдения, определить прогностически значимые факторы, влияющие на выживаемость больных с АКТГ-эктопированным синдромом (АКТГ-ЭС).

МАТЕРИАЛЫ И МЕТОДЫ: ретроспективное обсервационное одноцентровое одномоментное исследование 142 пациентов (86 женщин, 56 мужчин) с установленным в ФГБУ «НМИЦ Эндокринологии» Минздрава России клиническим диагнозом АКТГ-ЭС в период с 1990 по май 2021 гг. Проанализированы демографические сведения о пациентах, данные анамнеза, результаты гормональных и инструментальных исследований на до- и послеоперационных этапах и отдаленные результаты лечения. Статистическая обработка данных проводилась при помощи пакета статистических программ IBM SPSS Statistics 23.

РЕЗУЛЬтАТЫ: возраст пациентов на момент диагностики заболевания составил от 12 до 76 лет (Ме 40 лет [28;54]). Длительность заболевания с момента появления симптомов до верификации диагноза — от 2 до 168 мес (Ме 20,5 мес [8;46]). Циклическое течение зафиксировано у 11 больных (7,7\%). В 86 случаях (60,6\%) первичный очаг локализовался в легком, в 13 - в средостении, в 7 - в поджелудочной железе, в 5 - в надпочечнике, по 1 случаю - в слепой кишке и червеобразном отростке, у 1 - МРЩЖ, у $28(19,7 \%)-$ НЭО неустановленной локализации. Среди осложнений в активной стадии заболевания преобладали АГ ( $n=120(84,5 \%))$, остеопороз ( $n=87$ (61,3\%)), СД ( $n=78(54,9 \%))$, CС3 ( $n=75$ (52,8\%)). Ме периода наблюдения составила 36 мес [11;68,3], максимально 379 мес. Длительность периода наблюдения пациентов с неустановленным первичном очагом варьировала от 1 до 134 мес (Mе 16,5 мес [9,5;36,3]). На момент исследования первичный очаг удален у 90 больных (63,4\%), двусторонняя адреналэктомия по жизненным показаниям выполнена 24 пациентам (16,9\%), 17 из них с НЭО неустановленной локализации, 7 - с целью контроля тяжелого течения гиперкортицизма после радикального хирургического лечения. Метастазы выявлены у 29 (20,4\%). На момент последнего наблюдения $58(64,4 \%)$ - в ремиссии заболевания, у $12(13,3 \%)$ - рецидив, 37 (26,1\%) умерли от полиорганной недостаточности $(\mathrm{n}=20)$, ТЭЛА ( $\mathrm{n}=5)$, сердечно-сосудистых событий (ОНМK $(\mathrm{n}=2)$, внезапная сердечная смерть $(\mathrm{n}=2))$, осложнений хирургического вмешательства $(n=2)$, ДВС-синдрома $(n=1)$, перелома шейки бедра $(n=1)$, COVID-19 $(n=2)$, в 2 случаях причина летального исхода неизвестна. Многофакторный регрессионный анализ по методу Кокса показал, что значимое влияние на выживаемость больных оказывают возраст установки диагноза (OR 1,040; 95\% ДИ 1,014-1,068; $\mathrm{p}=0,003)$, локализация НЭО в легком (OR 0,294; 95\% ДИ 0,129-0,671; p=0,004), наличие метастазов (OR 3,413; 95\% ДИ 1,531-7,610; $p=0,003)$.

Выводы: наиболее частым источником эктопической продукции АКТГ являются НЭО бронхолегочной локализации (60,6\% случаев). Радикальное хирургическое лечение в 64,4\% случаев позволяет добиться стойкой ремиссии заболевания; рецидивы возникают в 13,3\% случаев. Возраст установки диагноза, локализация НЭО, наличие метастазов являются значимыми прогностическими факторами, влияющими на выживаемость больных.

КЛЮЧЕВЫЕ СЛОВА: АКТГ-эктопированный синдром; нейроэндокринная опухоль (НЭО); гиперкортичизм; карциноид легкого; карииноид тимуса; хирургическое лечение; отдаленные результаты. 